\title{
Antimicrobial-impregnated and -coated shunt catheters for prevention of infections in patients with hydrocephalus: a systematic review and meta-analysis
}

\author{
Athanasios A. Konstantelias, MD, ${ }^{1}$ Konstantinos Z. Vardakas, MD, PhD, ${ }^{1,2}$ \\ Konstantinos A. Polyzos, MD, MSc, ${ }^{1}$ Giannoula S. Tansarli, MD, ${ }^{1}$ \\ and Matthew E. Falagas, MD, MSc, DSc ${ }^{1-3}$
}

${ }^{1}$ Alfa Institute of Biomedical Sciences; ${ }^{2}$ Department of Medicine-Infectious Diseases, laso General Hospital, laso Group, Athens, Greece; and ${ }^{3}$ Tufts University School of Medicine, Boston, Massachusetts

\begin{abstract}
OBJECT The aim of this study was to evaluate the effectiveness of antimicrobial-impregnated and -coated shunt catheters (antimicrobial catheters) in reducing the risk of infection in patients undergoing CSF shunting or ventricular drainage.

METHODS The PubMed and Scopus databases were searched. Catheter implantation was classified as either shunting (mainly ventriculoperitoneal shunting) or ventricular drainage (mainly external [EVD]). Studies evaluating antibioticimpregnated catheters (AICs), silver-coated catheters (SCCs), and hydrogel-coated catheters (HCCs) were included. A random effects model meta-analysis was performed.
\end{abstract}

RESULTS Thirty-six studies ( 7 randomized and 29 nonrandomized, 16,796 procedures) were included. The majority of data derive from studies on the effectiveness of AICs, followed by studies on the effectiveness of SCCs. Statistical heterogeneity was observed in several analyses. Antimicrobial shunt catheters (AICs, SCCs) were associated with lower risk for CSF catheter-associated infections than conventional catheters (CCs) (RR $0.44,95 \% \mathrm{Cl} 0.35-0.56$ ). Fewer infections developed in the patients treated with antimicrobial catheters regardless of randomization, number of participating centers, funding, shunting or ventricular drainage, definition of infections, de novo implantation, and rate of infections in the study. There was no difference regarding gram-positive bacteria, all staphylococci, coagulase-negative streptococci, and Staphylococcus aureus, when analyzed separately. On the contrary, the risk for methicillin-resistant $S$. aureus (MRSA, RR 2.64, 95\% Cl 1.26-5.51), nonstaphylococcal (RR 1.75, 95\% Cl 1.22-2.52), and gram-negative bacterial (RR $2.13,95 \% \mathrm{Cl} 1.33-3.43$ ) infections increased with antimicrobial shunt catheters.

CONCLUSIONS Based on data mainly from nonrandomized studies, AICs and SCCs reduce the risk for infection in patients undergoing CSF shunting. Future studies should evaluate the higher risk for MRSA and gram-negative infections. Additional trials are needed to investigate the comparative effectiveness of the different types of antimicrobial catheters. http://thejns.org/doi/abs/10.3171/2014.12.JNS14908

KEY WORDS cerebrospinal shunt; antibiotic-impregnated catheters; silver-coated shunt; hydrogel-catheters; ventriculoperitoneal shunt; external ventricular drainage; hydrocephalus; infection

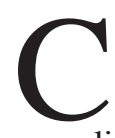

EREBROSPINAL fluid (CSF) shunting is a commonly used surgical procedure for the treatment of hydrocephalus. Infections are among the most common complications following shunt implantation, occurring after $5 \%$ to $15 \%$ of procedures, and they have been associated with increased morbidity, including lower intellectual ability, higher number of revision surgeries, prolonged hos- pitalization, and higher cost. ${ }^{4,15,49}$ In addition, CSF shunt infections were identified as predictors of mortality, which in such cases ranges from $1.5 \%$ to $22 \%$ in individual studies. ${ }^{4}$ Skin flora, including Staphylococcus spp., in up to $70 \%$ of cases and gram-negative bacilli (Escherichia coli, Klebsiella spp.) in up to $20 \%$ of cases are the main causative pathogens in CSF shunt infections. ${ }^{15}$ In CSF shunting, the risk

ABBREVIATIONS $\quad$ AIC $=$ antibiotic-impregnated catheter; $C C=$ conventional catheter; $C$ oNS $=$ coagulase-negative streptococci; $C S F=$ cerebrospinal fluid; $E V D=$ external ventricular drainage; $\mathrm{HCC}=$ hydrogel-coated catheter; $\mathrm{MRSA}=$ methicillin-resistant Staphylococcus aureus; $\mathrm{RCT}$ = randomized controlled trial; SCC = silver-coated catheter.

SUBMITTED April 23, 2014. ACCEPTED December 18, 2014.

INCLUDE WHEN CITING Published online March 13, 2015; DOI: 10.3171/2014.12.JNS14908.

DISCLOSURE The authors report no conflict of interest concerning the materials or methods used in this study or the findings specified in this paper. 
factors for shunt infections were younger age, including neonatal period and age less than 6 months, prematurity, and postoperative CSF leakage. The identified risk factors for ventricular drainage were previous shunt insertion, a duration of ventriculostomy for more than 5 days, previous craniotomy, and the etiology of hydrocephalus (including intraventricular and subarachnoid hemorrhage). ${ }^{15}$

Several efforts have been made to reduce the incidence of CSF catheter implantation-associated infections, such as using minimal handling protocols, including changing gloves before handling the catheter and laparoscopic placement of the distal catheter instead of conventional laparotomy. ${ }^{32,38}$ Perioperative antibiotic prophylaxis, antibiotic-impregnated sutures for wound closing, and topical application of methicillin to the operative field have also been shown to reduce infections associated with CSF catheter implantation. ${ }^{37,42,45}$ The development of antimicrobial-impregnated and -coated catheters (antimicrobial catheters)-including antibiotic-impregnated catheters (AICs), silver-coated catheters (SCCs), and hydrogel-coated catheters (HCCs) - has been among the most promising advances. AICs contain a combination of antibioticsrifampin with either clindamycin or minocycline-which are released through the lumen after the placement of the catheter. ${ }^{1}$ SCCs contain a combination of metallic silver and an insoluble silver salt that is also released through the lumen of the catheter. ${ }^{12}$ HCCs have a hydrophilic surface that impedes bacterial adherence. ${ }^{22,24}$

Although their use has increased over time and they have virtually replaced conventional catheters (CCs) in daily practice, ${ }^{16,24,27}$ the effectiveness of antimicrobial catheters in reducing CSF catheter implantation infections remains controversial. ${ }^{20,25,33,46,50}$ Additional topics in debate are whether antimicrobial catheters reduce the risk of infection in lower risk populations (for example adults rather than children), their impact on mortality, and whether they prevent colonization. We conducted a systematic review and meta-analysis to study the effectiveness of antimicrobial catheters in preventing CSF shunting-associated infections.

\section{Methods}

\section{Search Strategy, Study Selection, and Data Extraction}

Two investigators (A.A.K. and K.A.P.) conducted a systematic search of the PubMed and Scopus electronic databases using the following search terms: "antibiotic OR antimicrobial AND (cerebrospinal fluid OR ventriculoperitoneal OR external ventricular drainage) AND (shunt OR catheter) AND infection." Additional searches were performed with the keywords: "antibiotic AND shunt", "external AND ventricular AND drainage", "silver AND shunt", "silver AND CSF", "hydrogel AND shunt", "hydrogel AND CSF". The 2 investigators performed a separate, independent search of the literature and extracted relevant data. If discrepancies occurred, they were resolved in meetings with a third investigator (K.Z.V.). We also reviewed the references of the primarily retrieved studies to identify additional potentially eligible studies. The final search was done in January 2014. There was no limit on the year of publication.
A study was eligible for inclusion in the meta-analysis if it met the following criteria: 1) it provided comparative data regarding the development of infection or mortality in patients with any type of antimicrobial and conventional CSF shunt catheters, 2) it was published in a peerreviewed journal, and 3) it was written in the English language. Both primary insertion and revision procedures were considered eligible surgical interventions. Randomized controlled trials (RCTs) and nonrandomized studies in adults, children, infants, or neonates were eligible. A study was excluded if: 1) no control group was defined, 2 ) it was a case report or included fewer than 10 patients, or 3) it was considered part of a bigger study (multiple publications). The extracted data included study design, geographic region, type of catheters, duration of follow-up period, funding, population characteristics, type of neurosurgical procedure, and outcomes (infection, mortality).

\section{Definitions and Outcomes}

The definition of shunt infection was based on the definition provided in each of the included studies. Shunting refers to ventriculoperitoneal, ventriculoatrial, ventriculopleural, lumboperitoneal, cystoperitoneal, and subdural shunting. Ventricular drainage included external ventricular drainage (EVD) and ventriculosubgaleal shunting. Early shunt infection for permanent shunting was defined as infection developing during the first 6 months after the shunt implantation. Late shunt infection for permanent shunting was defined as infection developing more than 6 months after the shunt implantation. The primary outcome of the meta-analysis was development of infections with antimicrobial catheters compared with CCs. Infections due to specific microbes and infection-related and all-cause mortality were considered secondary outcomes.

\section{Statistical Analysis}

Since it was expected that many nonrandomized studies were to be included in the analysis and significant clinical and possibly statistical heterogeneity was expected, the Mantel-Haenszel random effects model was applied for all analyses. Pooled risk ratios and 95\% confidence intervals were calculated regarding all outcomes. Statistical heterogeneity between studies was assessed by using the chi-square test $(\mathrm{p}<0.10$ was defined to indicate the presence of heterogeneity) and $\mathrm{I}^{2}$ (for assessing the degree of heterogeneity). Publication bias was assessed using the funnel-plot method. The meta-analysis was performed with Review Manager for Windows, version 5.2.

\section{Quality Assessment}

Quality assessment of the randomized and nonrandomized studies was performed with Jadad Scale ${ }^{18}$ and the Newcastle-Ottawa Scale, ${ }^{51}$ respectively.

\section{Results \\ Characteristics of the Included Studies}

We identified a total of 36 studies that evaluated 16,796 procedures eligible for systematic review and meta-analysis. ${ }^{1-3,5,6,11-14,16,17,19-24,26-31,34-36,39-41,43,44,52-56}$ The selection pro- 


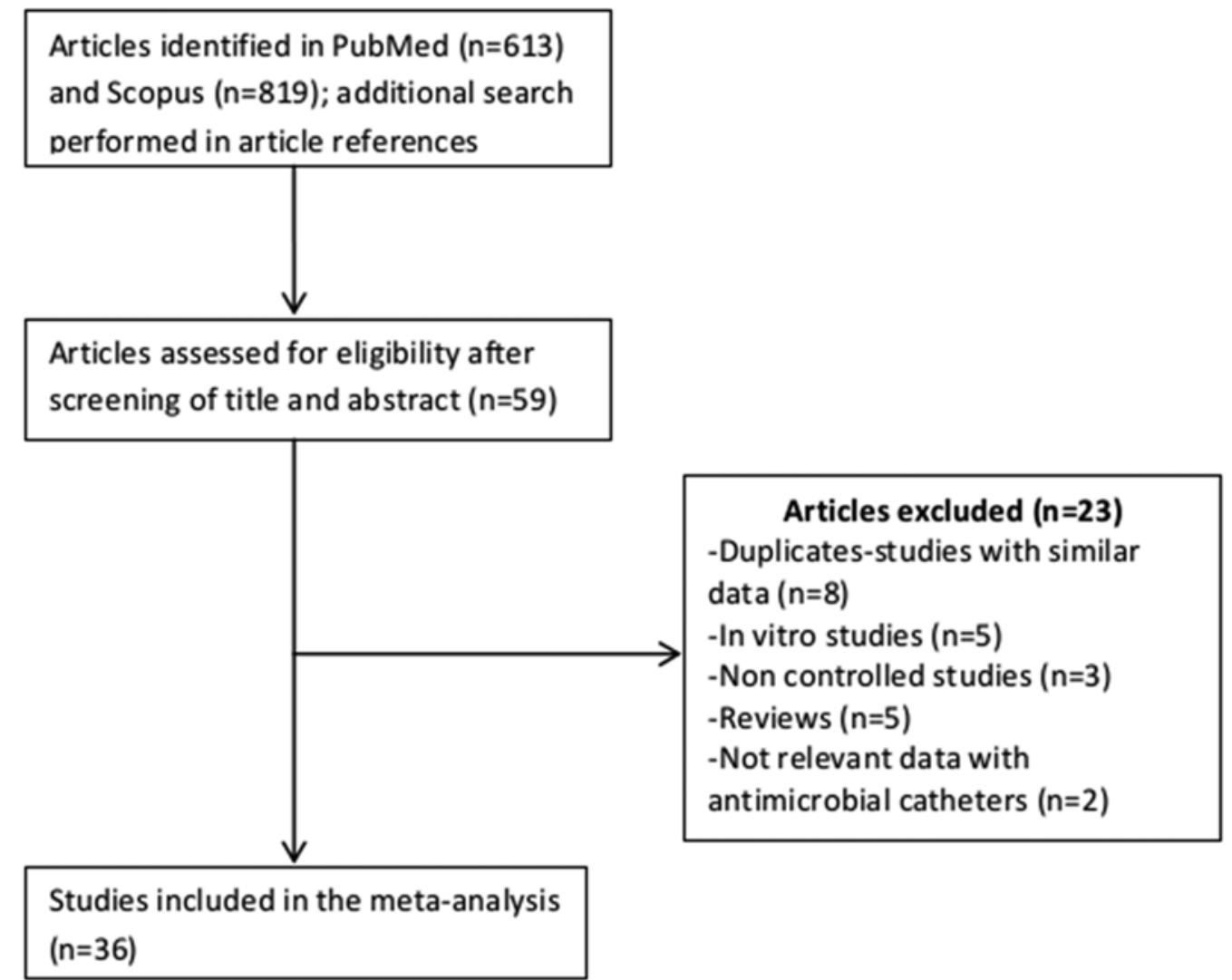

FIG. 1. Flow diagram of the selection process of the included studies.

cess is depicted in Fig. 1. The main characteristics of included studies are presented in Table 1. Twenty-nine studies were nonrandomized (19 retrospective and 10 prospective) and evaluated 15,335 procedures; 7 were randomized and evaluated 1461 procedures. The quality assessment of the included studies is presented in Appendix Tables 1 and 2. For RCTs, scores ranging from 2 to 5 (median 2) were calculated; for nonrandomized studies, the corresponding range was 5-9 (median 7). There were 27 single-center studies and 9 multicenter studies. Eighteen studies were conducted in Europe, 9 studies in the US, 2 in Africa, 1 in Canada, 1 in Asia, 1 in Australia, and 1 in New Zealand. Three studies were international. Eleven studies were funded either by the manufacturer of the catheter or an independent source.

In 22 studies, a positive culture was considered definitive for shunt infection while in 13 studies the diagnosis of infection was based on the presence of symptoms, signs of infection, laboratory findings in CSF and/or blood, and positive cultures. One study did not provide a definition for CSF shunt infection. The populations across and within the studies were characterized by heterogeneity regarding the proportion of the causes of hydrocephalus and the risk factors for shunt infection.

\section{Prevention of Infections}

The meta-analysis and subgroup analyses are presented in Table 2. . $-3,5,6,11-14,16,17,19-24,26-31,34-36,39-41,43,44,52-56$ Overall, the meta-analysis showed that the use of antimicrobial shunt catheters, after the exclusion of studies evaluating HCCs, was associated with lower risk for infection when compared with CCs $(15,949$ procedures, risk ratio [RR] $0.44,95 \%$ CI $0.35-0.56$, Fig. 2). Publication bias was detected, mainly in the region of small studies with negative results (Appendix Fig. 1). Fewer CSF infections developed in the antimicrobial catheter group regardless of randomization (Fig. 3), number of participating centers, funding (by the manufacturer or independent sources), shunting or ventricular drainage placement of the catheter, population age, definition of infections, and rate of infections in the study. The risk of infection was lower in de novo placement but not in revision surgery. There was no difference regarding gram-positive, staphylococcal, coagulasenegative streptococci (CoNS), and Staphylococcus aureus infections, separately. On the contrary, the risk for methicillin-resistant $S$. aureus (MRSA) infection was higher with antimicrobial catheters than CCs. Finally, patients treated with antimicrobial catheters had a higher risk for infection due to gram-negative bacteria and nonstaphylococcal species (data for a more detailed analysis according to specific pathogens were not available). No or minimal heterogeneity was observed in the analyses according to microbial etiology.

\section{Antibiotic-Impregnated Catheters}

Lower risk for infection was observed when AICs were compared with CCs for all types of CSF catheter implan-

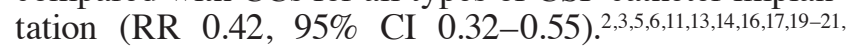




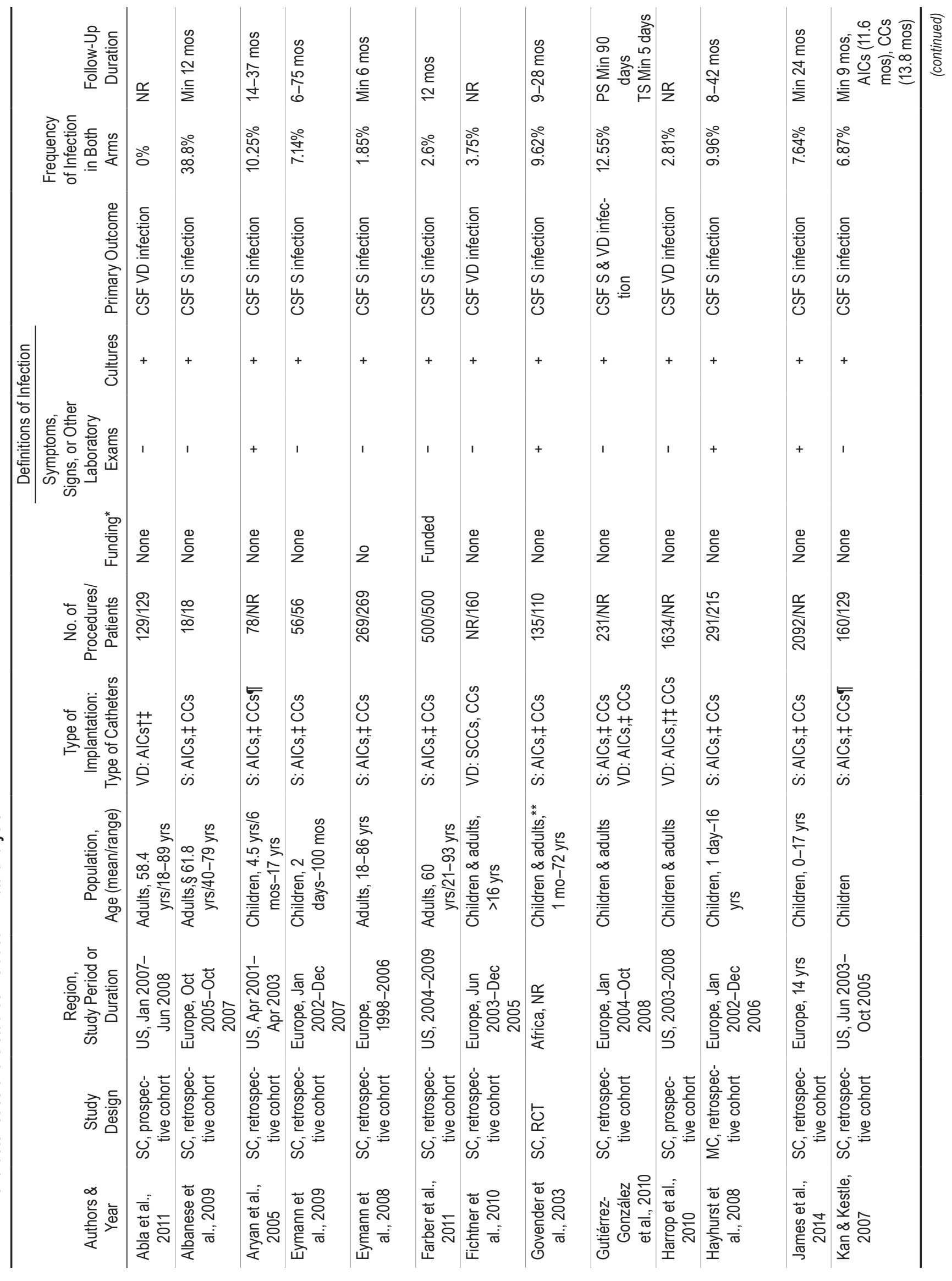




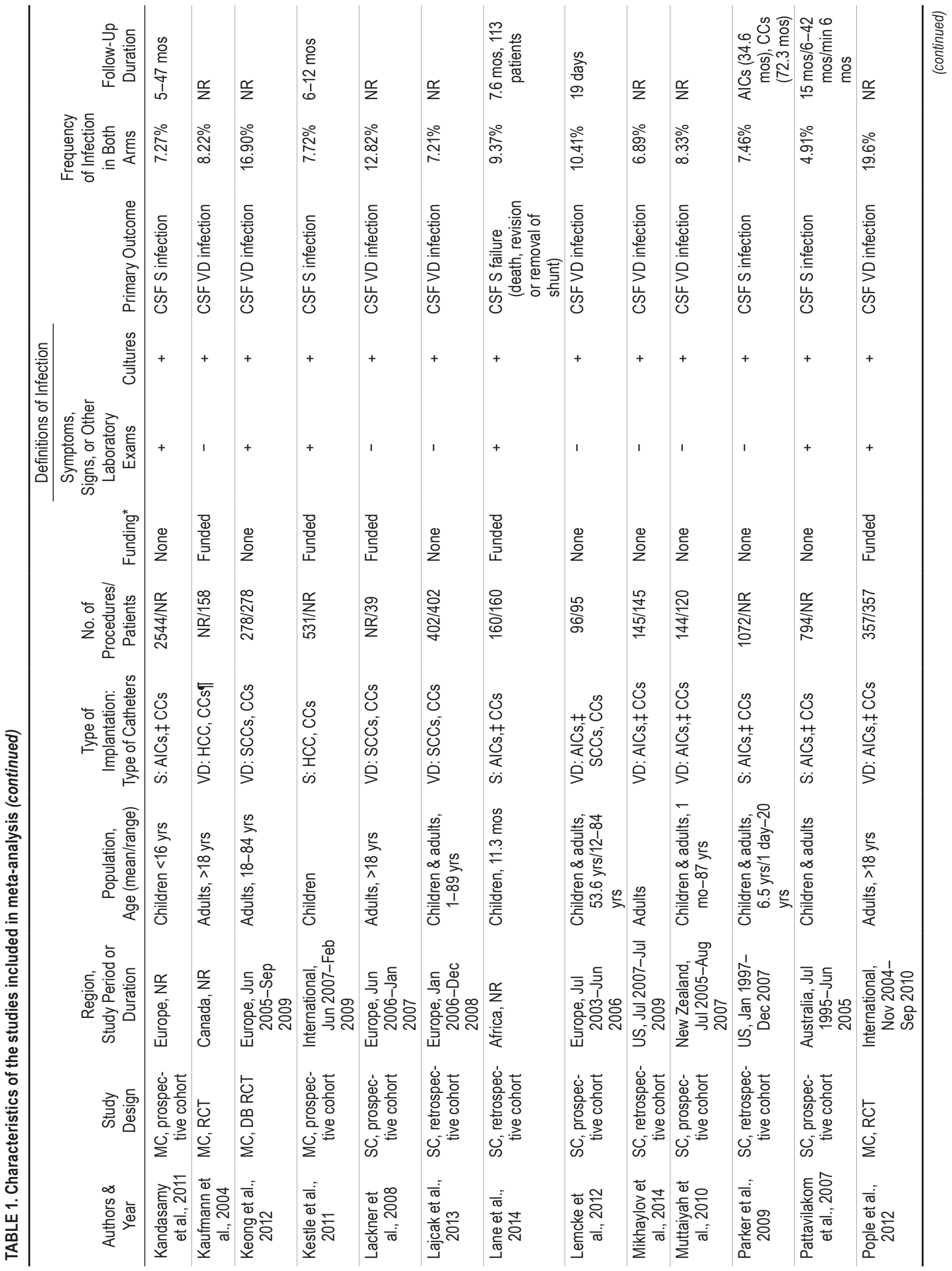




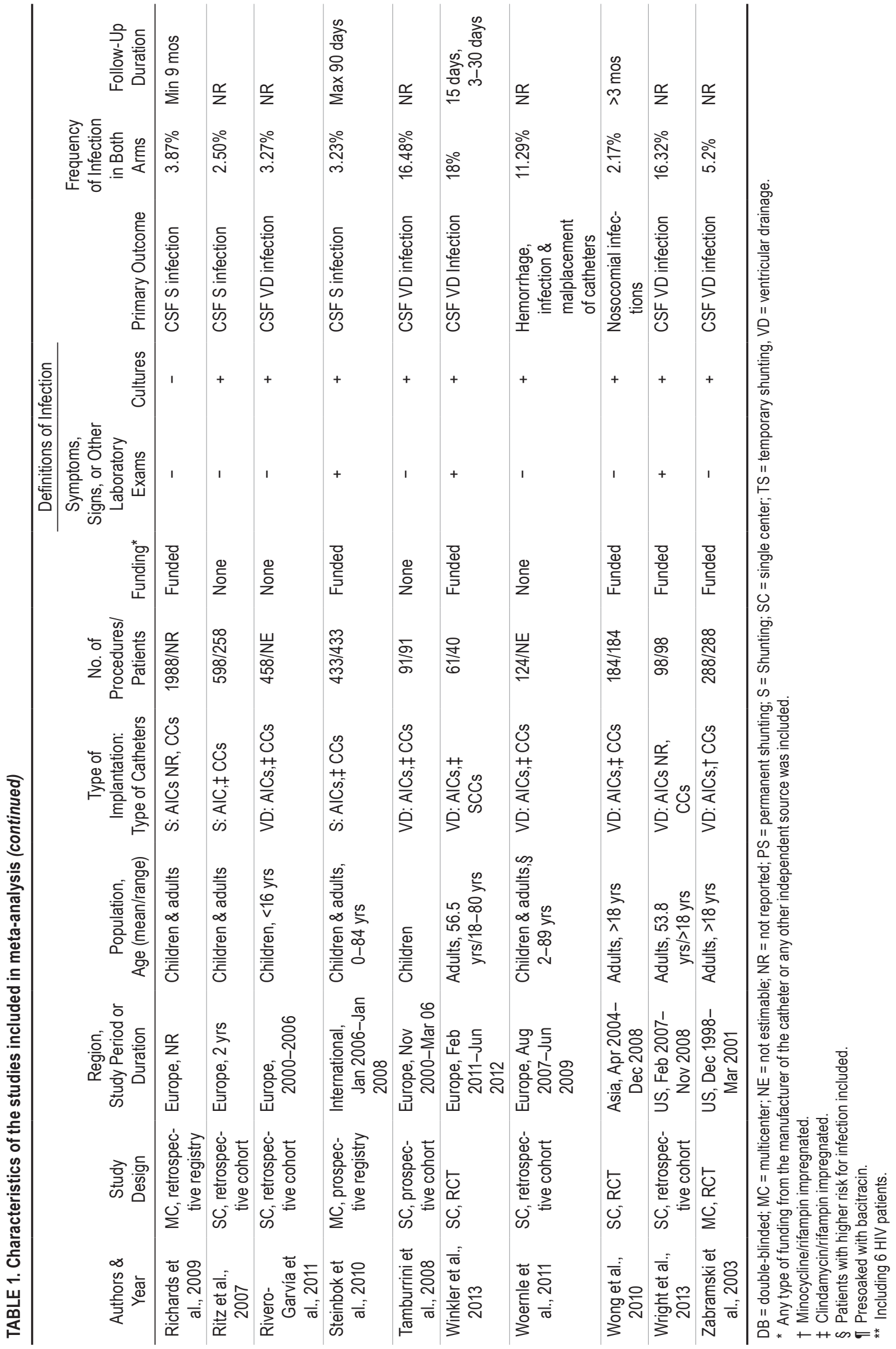


28-31,34-36,39-41,43,44,53-56 Subgroup analyses (Table 3) showed that AICs were associated with lower risk for infections regardless of randomization (for randomized studies, a trend toward a significant difference was observed), number of participating centers in the study, funding (by the manufacturer or independent sources), shunting or ventricular drainage, early-onset infections in permanent shunting, age of studied population, definition of infection, de novo catheter implantation, and rate of infection in the participating center(s). AICs were not associated with fewer infections in revision surgery in any type of implantation or late-onset infections in permanent shunting. After excluding from sensitivity analyses studies that had significant differences in their designs (studies were excluded because of the use of bacitracin-soaked catheters instead of CCs) and studies involving more than 1000 procedures, we found that the development of CSF shunt infections was also lower with AICs than with CCs. Finally, no difference was observed when rifampin-minocyclin impregnated catheters were compared with rifampin-clindamycin impregnated catheters (RR 1.14, 95\% CI 0.25-5.24). No difference was observed in risk for infections due to Staphylococcus spp. (regardless of the type of shunting), CoNS, S. aureus, or gram-positive bacteria, but the risk was higher for infection due to gram-negative bacteria, nonstaphylococcal species, and MRSA.

\section{Silver-Coated and Hydrogel-Coated Catheters}

Data regarding the comparative effectiveness of SCCs and CCs were available only for ventricular drainage catheter placement (Table 4). ${ }^{12,23,26,27,29}$ SCCs were associated with lower risk for infection compared with CCs (RR 0.60, 95\% CI 0.40-0.89). The difference was significant in the single multicenter, randomized trial (RR 0.57, 95\% CI 0.33-0.99) but not in the analysis of 4 single-center, nonrandomized studies. SCCs were associated with lower risk for infection in nonfunded studies and in center(s) with a high rate of infection ( $>10 \%$ ), but no difference was found between SCCs and CCs with regard to population age, definition of infection, low rate of infection $<10 \%$, or microbial etiology. Heterogeneity was not observed in these analyses.

Two multicenter studies (a randomized trial involving adults and a prospective cohort study involving children) evaluated HCCs compared with CCs for the prevention of shunt infections. ${ }^{22,24}$ HCCs were not associated with fewer infections than CCs (689 procedures, RR 1.63, 95\% CI $0.21-12.96)$.

\section{Silver-Coated Versus Antibiotic-Impregnated Catheters}

SCCs were compared with AICs for ventricular drainage in 2 studies (a randomized trial involving adults and a prospective cohort involving children and adults). ${ }^{29,52}$ No difference in the risk for infection was observed in either of these studies or the pooled analysis (RR 1.37, 95\% CI $0.55-3.41)$.

\section{Mortality}

Eleven studies provided data for all-cause mortality (1910 patients), ${ }^{1,2,12,13,23,26,28,30,43,44,54} 8$ studied AICs and 3
TABLE 2. Development of infections in subgroup analyses of all studies evaluating antimicrobial catheters

\begin{tabular}{|c|c|c|c|}
\hline $\begin{array}{c}\text { Studied } \\
\text { Characteristic }\end{array}$ & $\begin{array}{c}\text { No. of } \\
\text { Procedures }\end{array}$ & $\begin{array}{l}\text { No. of } \\
\text { Studies }\end{array}$ & $\operatorname{RR}(95 \% \mathrm{Cl}), 1^{2}$ \\
\hline All studies & 16,638 & 34 & $0.46(0.36-0.58), 48 \%$ \\
\hline Randomized studies & 1400 & 6 & $0.59(0.39-0.89), 27 \%$ \\
\hline $\begin{array}{l}\text { Nonrandomized } \\
\text { studies }\end{array}$ & 15,238 & 28 & $0.44(0.33-0.57), 50 \%$ \\
\hline Single-center studies & 9770 & 25 & $0.38(0.28-0.50), 41 \%$ \\
\hline Multicenter studies & 6868 & 9 & $0.68(0.54-0.86), 13 \%$ \\
\hline $\begin{array}{l}\text { Funded studies, } \\
\text { funded by any } \\
\text { source }\end{array}$ & 4736 & 11 & $0.50(0.33-0.77), 37 \%$ \\
\hline $\begin{array}{l}\text { Funded studies, } \\
\text { funded by manu- } \\
\text { facturer }\end{array}$ & 3607 & 8 & $0.58(0.40-0.85), 24 \%$ \\
\hline Nonfunded studies & 11,902 & 23 & $0.44(0.34-0.59), 52 \%$ \\
\hline Permanent (shunting) & 11,838 & 18 & $0.48(0.37-0.64), 33 \%$ \\
\hline $\begin{array}{l}\text { Temporary (ventricu- } \\
\text { lar drainage) }\end{array}$ & 4800 & 18 & $0.44(0.30-0.65), 60 \%$ \\
\hline Children & 6461 & 10 & $0.58(0.42-0.80), 23 \%$ \\
\hline Adults & 2334 & 11 & $0.43(0.28-0.67), 36 \%$ \\
\hline $\begin{array}{l}\text { Mixed adults \& } \\
\text { children }\end{array}$ & 7843 & 14 & $0.43(0.29-0.64), 61 \%$ \\
\hline $\begin{array}{l}\text { Clinical \& labora- } \\
\text { tory definition of } \\
\text { infection }\end{array}$ & 7791 & 12 & $0.58(0.45-0.76), 29 \%$ \\
\hline $\begin{array}{l}\text { Definition of infection } \\
\text { by cultures only }\end{array}$ & 7511 & 22 & $0.39(0.28-0.53), 41 \%$ \\
\hline $\begin{array}{l}\text { Rate of infection } \\
>10 \%\end{array}$ & 1442 & 10 & $0.46(0.29-0.73), 48 \%$ \\
\hline $\begin{array}{l}\text { Rate of infection } \\
5-10 \%\end{array}$ & 7738 & 11 & $0.55(0.40-0.75), 43 \%$ \\
\hline Rate of infection $<5 \%$ & 7458 & 13 & $0.36(0.23-0.57), 51 \%$ \\
\hline De novo implantation & 1820 & 9 & $0.50(0.33-0.74), 0 \%$ \\
\hline Revision implantation & 1908 & 3 & $0.83(0.55-1.26), 0 \%$ \\
\hline $\begin{array}{l}\text { Gram-positive infec- } \\
\text { tions }\end{array}$ & 441 & 20 & $0.95(0.83-1.08), 15 \%$ \\
\hline $\begin{array}{l}\text { Staphylococcal infec- } \\
\text { tions }\end{array}$ & 385 & 17 & $0.86(0.73-1.01), 0 \%$ \\
\hline CoNS infections & 305 & 16 & $0.83(0.58-1.19), 22 \%$ \\
\hline S. aureus infections & 342 & 12 & $1.02(0.79-1.31), 0 \%$ \\
\hline MRSA infections & 124 & 5 & $2.64(1.26-5.51), 0 \%$ \\
\hline $\begin{array}{l}\text { Nonstaphylococcal } \\
\text { infections }\end{array}$ & 371 & 14 & $1.75(1.22-2.52), 10 \%$ \\
\hline $\begin{array}{l}\text { Gram-negative infec- } \\
\text { tions }\end{array}$ & 412 & 16 & $2.13(1.33,3.43), 0 \%$ \\
\hline
\end{tabular}

studied SCCs, all in comparison with CCs. No data for mortality was available for HCCs. When all types of antimicrobial catheters were compared with CCs, no difference was observed in all-cause mortality (RR 1.17, 95\% CI $0.92-1.49$ ). Table 5 shows the subgroup analyses; no difference in mortality was seen for AICs compared with $\mathrm{CCs}$ for ventricular drainage or shunting and no difference 


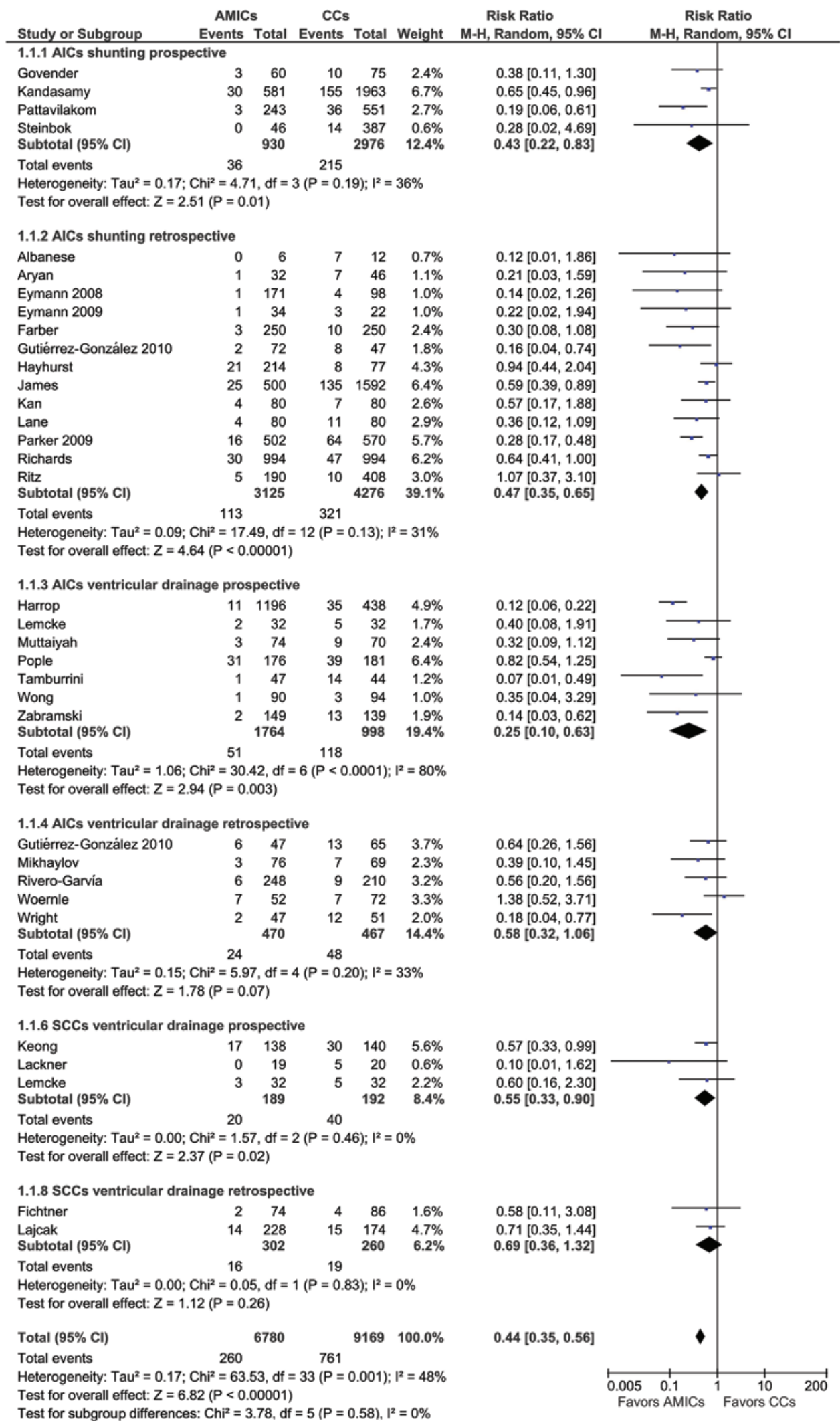

FIG. 2. Forest plot depicting the risk ratios for CSF shunting- and ventricular drainage-associated infections for antimicrobial catheters (AMICs [AICs and SCCs]) compared with CCs (studies on HCCs were not included in the figure). The vertical line indicates the "no difference" point between the 2 regimens; squares indicate risk ratios; diamonds, pooled risk ratios for all studies; horizontal lines, $95 \%$ confidence intervals; events represent numbers of infections; total refers to number of procedures. $\mathrm{M}-\mathrm{H}=$ Mantel Haenszel. Figure is available in color online only. 


\begin{tabular}{|c|c|c|c|c|c|c|c|c|}
\hline \multirow{2}{*}{$\frac{\text { Study or Subgroup }}{\text { 4.1.1 AICs Randomized }}$} & \multicolumn{2}{|c|}{ AMICs } & $\begin{aligned} & \text { CCs } \\
& \text { Events }\end{aligned}$ & Total & Weight & $\begin{array}{c}\text { Risk Ratio } \\
\text { M-H, Random, } 95 \% \mathrm{CI}\end{array}$ & \multicolumn{2}{|c|}{$\begin{array}{c}\text { Risk Ratio } \\
\text { M-H, Random, } 95 \% \mathrm{Cl}\end{array}$} \\
\hline & & & & & & & & \\
\hline Govender & 3 & 60 & 10 & 75 & $2.5 \%$ & $0.38[0.11,1.30]$ & & 1 \\
\hline Pople & 31 & 176 & 39 & 181 & $6.5 \%$ & $0.82[0.54,1.25]$ & & \\
\hline Wong & 1 & 90 & 3 & 94 & $1.0 \%$ & $0.35[0.04,3.29]$ & $\longrightarrow$ & \\
\hline $\begin{array}{l}\text { Zabramski } \\
\text { Subtotal (95\% Cl) }\end{array}$ & 2 & $\begin{array}{l}149 \\
475\end{array}$ & 13 & $\begin{array}{l}139 \\
489\end{array}$ & $1.9 \%$ & $0.14[0.03,0.62]$ & & \\
\hline Total events & 37 & & 65 & & 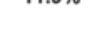 & 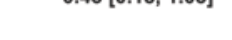 & & \\
\hline \multicolumn{9}{|c|}{ Heterogeneity: $\mathrm{Tau}^{2}=0.41 ; \mathrm{Ch}^{2}=6.53, \mathrm{df}=3(P=0.09) ; \mathrm{I}^{2}=54 \%$} \\
\hline \multicolumn{9}{|c|}{ 4.1.2 AICs Non-Randomized } \\
\hline Albanese & 0 & 6 & 7 & 12 & $0.7 \%$ & $0.12[0.01,1.86]$ & & \\
\hline Aryan & 1 & 32 & 7 & 46 & $1.1 \%$ & $0.21[0.03,1.59]$ & & \\
\hline Eymann 2008 & 1 & 171 & 4 & 98 & $1.0 \%$ & $0.14[0.02,1.26]$ & & \\
\hline Eymann 2009 & 1 & 34 & 3 & 22 & $1.0 \%$ & $0.22[0.02,1.94]$ & & \\
\hline Farber & 3 & 250 & 10 & 250 & $2.4 \%$ & $0.30[0.08,1.08]$ & & \\
\hline Gutiérrez-González 2010 & 8 & 119 & 21 & 112 & $4.3 \%$ & $0.36[0.17,0.78]$ & $\longrightarrow$ & \\
\hline Harrop & 11 & 1196 & 35 & 438 & $4.9 \%$ & $0.12[0.06,0.22]$ & & \\
\hline Hayhurst & 21 & 214 & 8 & 77 & $4.3 \%$ & $0.94[0.44,2.04]$ & & \\
\hline James & 25 & 500 & 135 & 1592 & $6.6 \%$ & $0.59[0.39,0.89]$ & & - \\
\hline Kan & 4 & 80 & 7 & 80 & $2.6 \%$ & $0.57[0.17,1.88]$ & & \\
\hline Kandasamy & 30 & 581 & 155 & 1963 & $6.8 \%$ & $0.65[0.45,0.96]$ & & $=$ \\
\hline Lane & 4 & 80 & 11 & 80 & $2.9 \%$ & $0.36[0.12,1.09]$ & & \\
\hline Lemcke & 2 & 32 & 5 & 32 & $1.8 \%$ & $0.40[0.08,1.91]$ & & \\
\hline Mikhaylov & 3 & 76 & 7 & 69 & $2.3 \%$ & $0.39[0.10,1.45]$ & & \\
\hline Muttaiyah & 3 & 74 & 9 & 70 & $2.4 \%$ & $0.32[0.09,1.12]$ & & \\
\hline Parker 2009 & 16 & 502 & 64 & 570 & $5.8 \%$ & $0.28[0.17,0.48]$ & - & \\
\hline Pattavilakom & 3 & 243 & 36 & 551 & $2.7 \%$ & $0.19[0.06,0.61]$ & C. & \\
\hline Richards & 30 & 994 & 47 & 994 & $6.3 \%$ & $0.64[0.41,1.00]$ & & - \\
\hline Ritz & 5 & 190 & 10 & 408 & $3.1 \%$ & $1.07[0.37,3.10]$ & & \\
\hline Rivero-Garvía & 6 & 248 & 9 & 210 & $3.2 \%$ & $0.56[0.20,1.56]$ & - & \\
\hline Steinbok & 0 & 46 & 14 & 387 & $0.6 \%$ & $0.28[0.02,4.69]$ & & \\
\hline Tamburrini & 1 & 47 & 14 & 44 & $1.2 \%$ & $0.07[0.01,0.49]$ & & \\
\hline Woernle & 7 & 52 & 7 & 72 & $3.3 \%$ & $1.38[0.52,3.71]$ & & \\
\hline $\begin{array}{l}\text { Wright } \\
\text { Subtotal }(95 \% \mathrm{Cl})\end{array}$ & 2 & $\begin{array}{r}47 \\
5814\end{array}$ & 12 & $\begin{array}{r}51 \\
8228\end{array}$ & $\begin{array}{r}2.0 \% \\
73.3 \%\end{array}$ & $\begin{array}{l}0.18[0.04,0.77] \\
0.41[0.31,0.55]\end{array}$ & 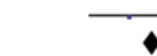 & \\
\hline Total events & 187 & & 637 & & & & & \\
\hline \multirow{2}{*}{\multicolumn{9}{|c|}{$\begin{array}{l}\text { Heterogeneity: } \mathrm{Tau}^{2}=0.22 ; \mathrm{Ch}^{2}=49.71, \mathrm{df}=23(\mathrm{P}=0.001) ; \mathrm{l}^{2}=54 \% \\
\text { Test for overall effect: } Z=6.05(P<0.00001)\end{array}$}} \\
\hline & & & & & & & & \\
\hline \multicolumn{9}{|l|}{ 4.1.3 SCCs Randomized } \\
\hline Keong & 17 & 138 & 30 & 140 & $5.7 \%$ & $0.57[0.33,0.99]$ & - & r \\
\hline Subtotal $(95 \% \mathrm{Cl})$ & & 138 & & 140 & $5.7 \%$ & $0.57[0.33,0.99]$ & & \\
\hline Total events & 17 & & 30 & & & & & \\
\hline \multirow{2}{*}{\multicolumn{9}{|c|}{$\begin{array}{l}\text { Heterogeneity: Not applicable } \\
\text { Test for overall effect: } Z=1.99(P=0.05)\end{array}$}} \\
\hline & & & & & & & & \\
\hline \multicolumn{9}{|c|}{ 4.1.4 SCCs Non-Randomized } \\
\hline Fichtner & 2 & 74 & 4 & 86 & $1.6 \%$ & $0.58[0.11,3.08]$ & & \\
\hline Lackner & 0 & 19 & 5 & 20 & $0.6 \%$ & $0.10[0.01,1.62]$ & & \\
\hline Lajcak & 14 & 228 & 15 & 174 & $4.7 \%$ & $0.71[0.35,1.44]$ & - & \\
\hline Lemcke & 3 & 32 & 5 & 32 & $2.2 \%$ & $0.60[0.16,2.30]$ & & \\
\hline Subtotal $(95 \% \mathrm{Cl})$ & & 353 & & 312 & $9.1 \%$ & $0.62[0.35,1.10]$ & & \\
\hline Total events & 19 & & 29 & & & & & \\
\hline \multirow{2}{*}{\multicolumn{9}{|c|}{$\begin{array}{l}\text { Heterogeneity: } \operatorname{Tau}^{2}=0.00 ; \mathrm{Ch}^{2}=1.92, \mathrm{df}=3(P=0.59) ; 1^{2}=0 \% \\
\text { Test for overall effect: } Z=1.63(P=0.10)\end{array}$}} \\
\hline & & & & & & & & \\
\hline Total $(95 \% \mathrm{Cl})$ & & 6780 & & 9169 & $100.0 \%$ & $0.44[0.35,0.56]$ & 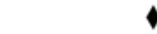 & \\
\hline Total events & 260 & & 761 & & & & & \\
\hline $\begin{array}{l}\text { Heterogeneity: } \operatorname{Tau}^{2}=0.1 \\
\text { Test for overall effect: } Z=\end{array}$ & $\begin{array}{l}\mathrm{Chi}^{2}=61.7 \\
84(\mathrm{P}<0 . \mathrm{C}\end{array}$ & $\begin{array}{l}78, \mathrm{df}= \\
00001)\end{array}$ & $32(P=0$ & 0.001); & $I^{2}=48 \%$ & & $\begin{array}{cc}0.005 & 0.1 \\
0 \text { Favors AMICs }\end{array}$ & $\begin{array}{c}1 \\
s \\
\text { Favors CCs }\end{array}$ \\
\hline
\end{tabular}

FIG. 3. Forest plot depicting the risk ratios for CSF shunting- and ventricular drainage-associated infections for antimicrobial catheters (AMICs [AICs and SCCs]) compared with CCs (studies on HCCs were not included in the figure). The vertical line indicates the "no difference" point between the 2 regimens; squares indicate risk ratios; diamonds, pooled risk ratios for all studies; horizontal lines, 95\% confidence intervals; events represent numbers of infections; total refers to number of procedures. Figure is available in color online only.

in mortality was seen for SCCs compared with CCs in ventricular drainage.

\section{Outcomes of Multivariate Analysis of Individual Studies}

Multivariate analyses were performed in 8 studies. Two studies showed that AICs were independently associated with lower risk for infection in permanent shunting ${ }^{13,14}$ while 2 others showed no difference between AICs and CCs. ${ }^{20,40}$ Two studies provided adjusted results in ventricular drainage; in one of them, AICs were independently associated with lower risk for infection, ${ }^{56}$ while in the second they were not. ${ }^{36}$ In the single study on SCCs that provided adjusted data, SCCs were associated with lower risk for infection. ${ }^{23}$ One study showed that HCCs were indepen- 
TABLE 3. Development of infections in subgroup analyses of studies with AICs

\begin{tabular}{|c|c|c|c|}
\hline $\begin{array}{c}\text { Studied } \\
\text { Characteristic }\end{array}$ & $\begin{array}{c}\text { No. of } \\
\text { Procedures }\end{array}$ & $\begin{array}{l}\text { No. of } \\
\text { Studies }\end{array}$ & $\mathrm{RR}(95 \% \mathrm{Cl}), \mathrm{I}^{2}$ \\
\hline All studies & 15,006 & 28 & $0.42(0.32-0.55), 54 \%$ \\
\hline Randomized studies & 964 & 4 & 0.43 (0.18-1.03), $54 \%$ \\
\hline $\begin{array}{l}\text { Nonrandomized } \\
\text { studies }\end{array}$ & 14,042 & 24 & $0.41(0.31-0.55), 54 \%$ \\
\hline Single-center studies & 9105 & 22 & $0.35(0.26-0.49), 44 \%$ \\
\hline Multicenter studies & 5901 & 6 & 0.68 (0.52-0.89), 21\% \\
\hline $\begin{array}{l}\text { Funded studies, } \\
\text { funded by any } \\
\text { source }\end{array}$ & 4008 & 8 & $0.47(0.30-0.73), 37 \%$ \\
\hline $\begin{array}{l}\text { Funded studies, } \\
\text { funded by manu- } \\
\text { facturer }\end{array}$ & 3410 & 6 & $0.57(0.37-0.86), 29 \%$ \\
\hline Nonfunded studies & 10,998 & 20 & $0.41(0.29-0.57), 58 \%$ \\
\hline Shunting & 11,307 & 17 & $0.48(0.37-0.62), 29 \%$ \\
\hline $\begin{array}{l}\text { Early infection in } \\
\text { permanent shunt }\end{array}$ & 1509 & 6 & 0.34 (0.13-0.88), $28 \%$ \\
\hline $\begin{array}{l}\text { Late infection in } \\
\text { permanent shunt }\end{array}$ & 807 & 4 & $1.23(0.24-6.20), 0 \%$ \\
\hline Ventricular drainage & 3699 & 12 & $0.36(0.20-0.64), 71 \%$ \\
\hline Neonates & 134 & 2 & $0.39(0.16-0.96), 0 \%$ \\
\hline Children & 5930 & 9 & $0.58(0.44-0.76), 12 \%$ \\
\hline Adults & 1859 & 8 & $0.32(0.16-0.63), 48 \%$ \\
\hline $\begin{array}{l}\text { Mixed adults \& } \\
\text { children }\end{array}$ & 7217 & 11 & 0.39 (0.24-0.63), 67\% \\
\hline $\begin{array}{l}\text { Clinical \& labora- } \\
\text { tory definition of } \\
\text { infection }\end{array}$ & 6982 & 10 & $0.57(0.42-0.76), 31 \%$ \\
\hline $\begin{array}{l}\text { Definition of infection } \\
\text { by cultures }\end{array}$ & 6688 & 20 & $0.34(0.24-0.49), 41 \%$ \\
\hline $\begin{array}{l}\text { Rate of infection } \\
>10 \%\end{array}$ & 1061 & 8 & $0.40(0.21-0.78), 60 \%$ \\
\hline $\begin{array}{l}\text { Rate of infection } \\
\quad 5-10 \%\end{array}$ & 6647 & 8 & $0.49(0.34-0.70), 47 \%$ \\
\hline Rate of infection $<5 \%$ & 7298 & 12 & $0.35(0.22-0.57), 54 \%$ \\
\hline De novo implantation & 1598 & 7 & $0.44(0.28-0.69), 0 \%$ \\
\hline Revision implantation & 1908 & 3 & $0.83(0.55-1.26), 0 \%$ \\
\hline $\begin{array}{l}\text { Gram-positive infec- } \\
\text { tions }\end{array}$ & 422 & 18 & 0.94 (0.82-1.09), 18\% \\
\hline $\begin{array}{l}\text { Staphylococcal infec- } \\
\text { tions }\end{array}$ & 366 & 15 & 0.87 (0.73-1.03), $0 \%$ \\
\hline CoNS infections & 286 & 14 & 0.86 (0.59-1.25), $27 \%$ \\
\hline S. aureus infections & 323 & 10 & $1.01(0.78-1.31), 0 \%$ \\
\hline MRSA infections & 119 & 4 & 2.64 (1.26-5.51), 0\% \\
\hline $\begin{array}{l}\text { Nonstaphylococcal } \\
\text { infections }\end{array}$ & 357 & 13 & 1.75 (1.16-2.65), 21\% \\
\hline $\begin{array}{l}\text { Gram-negative infec- } \\
\text { tions }\end{array}$ & 406 & 15 & 2.08 (1.29-3.37), $0 \%$ \\
\hline $\begin{array}{l}\text { Excluding studies w/ } \\
>1000 \text { procedures }\end{array}$ & 5676 & 23 & $0.43(0.32-0.59), 34 \%$ \\
\hline
\end{tabular}

(continued)
TABLE 3. Development of infections in subgroup analyses of studies with AICs (continued)

\begin{tabular}{cccc}
\hline $\begin{array}{c}\text { Studied } \\
\text { Characteristic }\end{array}$ & $\begin{array}{c}\text { No. of } \\
\text { Procedures }\end{array}$ & $\begin{array}{c}\text { No. of } \\
\text { Studies }\end{array}$ & RR $(95 \% \mathrm{Cl}), \mathrm{I}^{2}$ \\
\hline $\begin{array}{c}\text { Excluding studies } \\
\text { w/ bacitracin- } \\
\text { presoaked CCs }\end{array}$ & 14,768 & 26 & $0.42(0.32-0.55), 57 \%$ \\
\hline $\begin{array}{l}\text { Rifampin-clindamycin } \\
\text { vs rifampin-mino- } \\
\text { cyclin }\end{array}$ & 1325 & 2 & $1.14(0.25-5.24), \mathrm{NA}$ \\
\hline
\end{tabular}

NA = not applicable.

dently associated with higher risk for CSF shunting-associated infection. ${ }^{24}$

\section{Discussion}

This meta-analysis sought to investigate the protective effectiveness of antimicrobial catheters in reducing CSF shunting-associated infections in comparison with CCs. The majority of the included studies (28 studies, approximately $90 \%$ of included procedures) evaluated AICs, followed by SCCs; only 2 studies evaluated the effectiveness of HCCs. Antimicrobial catheters were associated with lower risk for infection compared with CCs regardless of randomization status, number of participating centers, funding, permanent or temporary catheter placement, de novo implantation, population age, timing of infection development, definition of infection, and rate of infections in the individual studies. Although no difference in the development of infections due to gram-positive bacteria, all staphylococci, CoNS, or S. aureus was observed, antimicrobial shunt catheters were associated with higher risk for MRSA, nonstaphylococcal, and gram-negative bacterial infections. It should be emphasized that only half of the included studies provided data regarding either specific bacterial species or gram-positive and gram-negative status.

Since the majority of the studies evaluated the effectiveness of AICs, the outcomes of the subgroup analyses regarding AICs were similar to that of the primary analysis. In addition, in a sensitivity analysis after the exclusion of studies with large populations (more than 1000 procedures), AICs were still associated with lower risk for infections. However, AICs were not more effective than CCs in randomized trials and in reducing the occurrence of late infections. Despite the trend toward lower risk for infection with the use of AICs in an analysis with a large sample size, the lack of statistical significance indicates that further studies are required to define the patient populations that would benefit more from this intervention. The presence of publication bias in the area of small trials with negative results is also an issue that needs to be addressed. The finding that AICs were not associated with prevention of late-onset infections (developing more than 6 months after catheter placement) in permanent shunting denotes that AICs exert their protective effectiveness during the first months after their implantation. However, it has been noted that early shunt infections account for approximately $70 \%$ of all episodes. ${ }^{2}$ Other interventions, and possibly 
TABLE 4. Development of infections in subgroup analyses of studies with SCC

\begin{tabular}{clccc}
\hline Compared Groups & \multicolumn{1}{c}{ Studied Characteristic } & No. of Patients & No. of Studies & RR $\left(95 \%\right.$ CI), $\left.\right|^{2}$ \\
\hline SCC vs CC & All studies & 943 & 5 & $0.60(0.40-0.89) 0 \%$ \\
\hline SCC vs CC & Nonrandomized studies & 665 & 4 & $0.62(0.35-1.10) 0 \%$ \\
\hline SCC vs CC & Single-center studies & 531 & 4 & $0.62(0.35-1.10) 0 \%$ \\
\hline SCC vs CC & Nonfunded studies & 904 & 4 & $0.62(0.42-0.92) 0 \%$ \\
\hline SCC vs CC & Ventricular drainage & 943 & 5 & $0.60(0.40-0.89) 0 \%$ \\
\hline SCC vs CC & Adults & 317 & 2 & $0.40(0.09-1.70) 36 \%$ \\
\hline SCC vs CC & Children \& adults & 626 & 3 & $0.67(0.38-1.20) 0 \%$ \\
\hline SCC vs CC & Definition of infection by cultures & 665 & 4 & $0.62(0.35-1.10) 0 \%$ \\
\hline SCC vs CC & Rate of infection >10\% & 381 & 3 & $0.55(0.33-0.90) 0 \%$ \\
\hline SCC vs CC & Rate of infection <10\% & 562 & 2 & $0.69(0.36-1.32) 0 \%$ \\
\hline SCC vs CC & Gram-positive infections & 19 & 3 & $0.88(0.49-1.58) 23 \%$ \\
\hline SCC vs CC & Staphylococcal infections & 19 & 3 & $0.68(0.34-1.37) 0 \%$ \\
\hline SCC vs CC & CoNS infections & 19 & 3 & $0.43(0.10-1.82) 0 \%$ \\
\hline SCC vs CC & S. aureus infections & 19 & 3 & $1.49(0.28-7.84) 6 \%$ \\
\hline SCC vs CC & Nonstaphylococcal infections & 14 & 2 & $2.67(0.46-15.48) 0 \%$ \\
\hline SCC vs AIC & Ventricular drainage & 125 & 2 & $1.37(0.55-3.41) 0 \%$ \\
\hline
\end{tabular}

stricter adherence to infection control measures and surgical techniques, are required to reduce the incidence of late-onset infections.

Five studies evaluated the effectiveness of SCCs for ventricular drainage; SCCs were associated with lower risk for infections in all studies and in the single available randomized trial. Overall, far fewer data were available for SCCs. It is possible that the non-statistically significant lower risk for development of infection in the SCC arm was due to the smaller sample size. However, it is noteworthy that statistical heterogeneity was not observed in these analyses. Limited data were also available for the comparative effectiveness of SCCs and AICs as well as of AICs with different antibiotics (rifampin/clindamycinimpregnated vs rifampin/minocycline-impregnated catheters). Although the antimicrobial spectrum of these combinations is quite similar for gram-positive bacteria, the comparative effectiveness could be evaluated in the future.

There are several concerns regarding the use of antimicrobial shunt catheters. The first is the cost-effectiveness of such an approach, which depends on the incidence of infections in an institution, the infection control measures, the surgical technique and expertise, the cost of catheters, and the cost of treatment of a possible subsequent infection in a given country. Klimo et al., in a cost-effectiveness analysis of AICs compared with CCs, concluded that the yearly cost savings from the use of AICs ranged from $\$ 90,000$ to over $\$ 1.3$ million in the US. ${ }^{25}$ In this analysis it was estimated that the total cost to treat a shunt infection accounted for up to $\$ 50,000$, while the additional cost of AICs compared with CCs was up to $\$ 400$ per kit. ${ }^{25}$ However, the authors acknowledged that in other countries where the cost to treat an infection is lower, the approach might not be cost-effective. Limited data regarding the cost of other types of antimicrobial shunt catheters did not allow a cost saving analysis compared with conventional catheters.

The second concern is the probability of a shift toward more virulent strains than CoNS. In this meta-analysis, AICs were associated with lower risk for any infection for both CSF shunting and ventricular drainage but higher risk for MRSA, nonstaphylococcal, and gram-negative bacterial infections. A large study performed in children showed that when CCs were used, CoNS were the predominant pathogen, accounting for approximately $52 \%$ of isolated pathogens, followed by S. aureus (31.6\%), Streptococcus or Enterococcus spp. (8.8\%), gram-negative organisms (4.4\%), and Propionibacterium acnes (2.2\%). When AICs replaced CCs, $S$. aureus became the predominant pathogen $(40 \%)$, followed by Streptococcus or Enterococcus

TABLE 5. Mortality in patients treated with CSF shunting or ventricular drainage

\begin{tabular}{llccc}
\hline \multicolumn{1}{c}{ Comparison } & Types of Catheters & No. of Procedures & No. of Studies & RR $\left(95 \%\right.$ Cl), $\left.\right|^{2}$ \\
\hline AICs \& SCCs vs CCs & All types & 1819 & 10 & $1.17(0.92-1.49), 0 \%$ \\
\hline AICs vs CCs & VD & 617 & 3 & $1.08(0.70-1.65), 41 \%$ \\
\hline AICs vs CCs & Shunting & 721 & 4 & $1.46(0.76-2.79), 0 \%$ \\
\hline AICs vs CCs & All types & 1331 & 7 & $1.17(0.87-1.56), 0 \%$ \\
\hline SCCs vs CCs & VD & 481 & 3 & $1.17(0.76-1.81), 0 \%$ \\
\hline
\end{tabular}


spp. (20\%), P. acnes and CoNS (both 16\%), and gram-negative organisms (4\%). ${ }^{19}$ Although we were not able to study in depth the reasons behind this finding, one could assume that this is probably due to inactivity of antibiotics used in AICs against such bacteria. Nosocomial MRSA strains are probably not susceptible to rifampin, minocycline, and clindamycin in most settings, while Pseudomonas aeruginosa strains are definitively not susceptible. Similarly, the susceptibility of Acinetobacter spp. and multidrugresistant Enterobacteriaceae to the aforementioned antibiotics is expected to be low. This shift toward more virulent pathogens than CoNS is an issue that warrants further study, since few of the included studies provided data for these comparisons, especially in settings where the incidence of MRSA or multidrug-resistant gram-negative bacteria is high. In addition, the impact of this shift on mortality should be explored.

The third issue is development of infections in patients who require replacement of catheters. Data regarding development of infections after de novo implantation showed that antimicrobial catheters reduce the risk for infections, but no difference was observed after revision implantation. Moreover, 1 study showed that patients requiring revision surgery with AICs who had an AIC implanted during the first operation had a higher infection rate (11.7\%) than those undergoing primary AIC insertion (1.6\%) and those undergoing revision of CCs using AICs (2.5\%). ${ }^{19}$

The meta-analysis showed no difference in all-cause mortality between patients treated with antimicrobial or conventional shunt catheters. Some might argue that for an infection with considerable mortality, the intervention could not be considered successful. However, we should bear in mind that antimicrobial catheters are used for prevention and not for treatment of infections. Factors such as the primary offending organism (CoNS in CSF shunt infections, which are less virulent than other bacteria), the antibiotics or other antimicrobials used in manufacturing the catheters, rates of mortality (provided in only 11 of the 36 included studies, range $0 \%-23.3 \%$ ) and infection (range $0 \%-38 \%$ in the included studies) in the medical center(s) where the study was performed, the time end point used in every study (which ranged from 3 days up to 7 years), and all-cause or infection-related mortality all deserve significant attention. Several meta-analyses in other fields of infectious diseases have failed to show a difference in mortality after the implementation of a preventive measure, despite a significant reduction in infection rate. ${ }^{7-10,47,48}$

To our knowledge, 4 meta-analyses have been published thus far. ${ }^{25,33,46,50}$ They included patients with ventricular drainage or shunt catheter implantation and concluded that antimicrobial catheters were more effective than CCs in preventing shunt infections. Besides the addition of recently published data, the present analysis outweighs the former ones for several other reasons. First, it included a greater number of studies (36 compared with $19,{ }^{46} 11,{ }^{33} 14,{ }^{25}$ and $8^{50}$ ) and consequently procedures (almost 17,000 compared with $6171,{ }^{46} 1649,339049,{ }^{25}$ and $2991^{50}$ ). It included more randomized trials ( 7 compared with $3,{ }^{46} 1,{ }^{16} 1,{ }^{18}$ and $\left.4^{50}\right)$, thus increasing the quality of its outcomes, and showed that randomized and nonrandomized studies have produced similar outcomes in most of the performed analyses. Several subgroup analyses were performed to study the heterogeneity (either statistically proven or suspected due to the different populations included) and provided support for further studies. The previous meta-analyses studied mainly the effects of age, number of participating centers, gram-positive or gramnegative bacteria, and study design on the development of infections. This meta-analysis also evaluated the effects of randomization, shunting or ventricular drainage catheter placement, funding, staphylococcal and nonstaphylococcal species, gram-positive and gram-negative bacteria, CoNS, S. aureus and MRSA, timing of infection development, clinical or microbiological diagnosis, the effectiveness of different types of antimicrobial catheters, and rates of infection in the participating centers. However, the majority of the data referred to AICs, and a lot fewer data were available for SCCs and HCCs. Finally, this meta-analysis provides a comprehensive review regarding the use of antimicrobial shunt catheters.

The most important limitation of the present meta-analysis is that it included mainly nonrandomized studies. Although residual confounding that could have affected the outcomes of the meta-analysis of nonrandomized studies cannot be ruled out, the similar findings in the subgroup analyses of randomized (although a marginally nonsignificant difference was observed in AICs probably due to smaller sample size) and nonrandomized studies reduce this possibility. Second, both clinical heterogeneity and statistical heterogeneity were present. Statistical heterogeneity ranged from 0 (no heterogeneity) up to $67 \%$ (considerable heterogeneity), even in subgroup analyses. The patients' demographic and clinical characteristics (including age and the etiology of hydrocephalus), the definition of shunt infection, and perioperative prophylaxis varied in the included studies; few of them provided adjusted results and therefore a meta-analysis of adjusted data were not feasible. Furthermore, ventricular drainage and shunt catheter placements are different surgical interventions that are associated with infections with different microbial etiology. The main cause of shunt-related infections is contamination due to skin flora during surgery, while the main cause of the ventricular drainage-related infections is retrograde colonization of the distal part of the catheter. ${ }^{15}$ Nevertheless, subgroup analyses did not show differences in outcomes of ventricular drainage and shunting. Finally, the differences in infection rate among the studies reflect the differences in the definition of shunt-associated infections as well as differences in infection control and surgical techniques or expertise.

\section{Conclusions}

Based mainly on data from nonrandomized, singlecenter, retrospective studies, this meta-analysis showed that the use of antimicrobial shunt catheters reduce the risk for CSF infections in patients with hydrocephalus. Several subgroup analyses showed that factors related to study design, type of catheter, duration of catheter placement, and whether the procedure is a de novo implantation or a revision may affect this risk. Publication bias in the region of small negative trials was also observed. In 
addition, a higher risk for infections due to more virulent bacteria than CoNS, namely MRSA and gram-negative bacilli, was observed. Due to the small number of studies providing data on such infections in the meta-analysis, this issue warrants further study. The choice as to whether antimicrobial catheters will be employed in an institution depends on both medical and financial variables. Finally, more data regarding the comparative effectiveness of AICs and SCCs, especially on the selection of specific bacterial species, is needed.

\section{References}

1. Abla AA, Zabramski JM, Jahnke HK, Fusco D, Nakaji P: Comparison of two antibiotic-impregnated ventricular catheters: a prospective sequential series trial. Neurosurgery 68:437-442, 2011

2. Albanese A, De Bonis P, Sabatino G, Capone G, Marchese E, Vignati A, et al: Antibiotic-impregnated ventriculo-peritoneal shunts in patients at high risk of infection. Acta Neurochir (Wien) 151:1259-1263, 2009

3. Aryan HE, Meltzer HS, Park MS, Bennett RL, Jandial R, Levy ML: Initial experience with antibiotic-impregnated silicone catheters for shunting of cerebrospinal fluid in children. Childs Nerv Syst 21:56-61, 2005

4. Choksey MS, Malik IA: Zero tolerance to shunt infections: can it be achieved? J Neurol Neurosurg Psychiatry 75:8791, 2004

5. Eymann R, Chehab S, Strowitzki M, Steudel WI, Kiefer M: Clinical and economic consequences of antibiotic-impregnated cerebrospinal fluid shunt catheters. J Neurosurg Pediatr 1:444-450,2008

6. Eymann R, Steudel WI, Kiefer M: Infection rate with application of an antibiotic-impregnated catheter for shunt implantation in children - a retrospective analysis. Klin Padiatr 221:69-73, 2009

7. Falagas ME, Fragoulis K, Bliziotis IA, Chatzinikolaou I: Rifampicin-impregnated central venous catheters: a metaanalysis of randomized controlled trials. J Antimicrob Chemother 59:359-369, 2007

8. Falagas ME, Siempos II: Prevention of ventilator-associated pneumonia: possible role of antimicrobials administered via the respiratory tract. Eur Respir J 31:1138-1139, 2008

9. Falagas ME, Siempos II, Bliziotis IA, Michalopoulos A: Administration of antibiotics via the respiratory tract for the prevention of ICU-acquired pneumonia: a meta-analysis of comparative trials. Crit Care 10:R123, 2006

10. Falagas ME, Vardakas KZ, Samonis G: Decreasing the incidence and impact of infections in neutropenic patients: evidence from meta-analyses of randomized controlled trials. Curr Med Res Opin 24:215-235, 2008

11. Farber SH, Parker SL, Adogwa O, McGirt MJ, Rigamonti D: Effect of antibiotic-impregnated shunts on infection rate in adult hydrocephalus: a single institution's experience. Neurosurgery 69:625-629, 2011

12. Fichtner J, Güresir E, Seifert V, Raabe A: Efficacy of silverbearing external ventricular drainage catheters: a retrospective analysis. J Neurosurg 112:840-846, 2010

13. Govender ST, Nathoo N, van Dellen JR: Evaluation of an antibiotic-impregnated shunt system for the treatment of hydrocephalus. J Neurosurg 99:831-839, 2003

14. Gutiérrez-González R, Boto GR, Fernández-Pérez C, del Prado N: Protective effect of rifampicin and clindamycin impregnated devices against Staphylococcus spp. infection after cerebrospinal fluid diversion procedures. BMC Neurol 10:93, 2010

15. Gutiérrez-González R, Boto GR, Pérez-Zamarrón A: Cerebrospinal fluid diversion devices and infection. A comprehensive review. Eur J Clin Microbiol Infect Dis 31:889-897, 2012
16. Harrop JS, Sharan AD, Ratliff J, Prasad S, Jabbour P, Evans JJ, et al: Impact of a standardized protocol and antibioticimpregnated catheters on ventriculostomy infection rates in cerebrovascular patients. Neurosurgery 67:187-191, 2010

17. Hayhurst C, Cooke R, Williams D, Kandasamy J, O'Brien DF, Mallucci CL: The impact of antibiotic-impregnated catheters on shunt infection in children and neonates. Childs Nerv Syst 24:557-562, 2008

18. Jadad AR, Moore RA, Carroll D, Jenkinson C, Reynolds DJ, Gavaghan DJ, et al: Assessing the quality of reports of randomized clinical trials: is blinding necessary? Control Clin Trials 17:1-12, 1996

19. James G, Hartley JC, Morgan RD, Ternier J: Effect of introduction of antibiotic-impregnated shunt catheters on cerebrospinal fluid shunt infection in children: a large single-center retrospective study. J Neurosurg Pediatr 13:101-106, 2014

20. Kan P, Kestle J: Lack of efficacy of antibiotic-impregnated shunt systems in preventing shunt infections in children. Childs Nerv Syst 23:773-777, 2007

21. Kandasamy J, Dwan K, Hartley JC, Jenkinson MD, Hayhurst C, Gatscher S, et al: Antibiotic-impregnated ventriculoperitoneal shunts-a multi-centre British paediatric neurosurgery group (BPNG) study using historical controls. Childs Nerv Syst 27:575-581, 2011

22. Kaufmann AM, Lye T, Redekop G, Brevner A, Hamilton M, Kozey M, et al: Infection rates in standard vs. hydrogel coated ventricular catheters. Can J Neurol Sci 31:506-510, 2004

23. Keong NC, Bulters DO, Richards HK, Farrington M, Sparrow OC, Pickard JD, et al: The SILVER (Silver Impregnated Line Versus EVD Randomized trial): a double-blind, prospective, randomized, controlled trial of an intervention to reduce the rate of external ventricular drain infection. Neurosurgery 71:394-404, 2012

24. Kestle JR, Riva-Cambrin J, Wellons JC III, Kulkarni AV, Whitehead WE, Walker ML, et al: A standardized protocol to reduce cerebrospinal fluid shunt infection: the Hydrocephalus Clinical Research Network Quality Improvement Initiative. J Neurosurg Pediatr 8:22-29, 2011

25. Klimo P Jr, Thompson CJ, Ragel BT, Boop FA: Antibioticimpregnated shunt systems versus standard shunt systems: a meta- and cost-savings analysis. J Neurosurg Pediatr 8:600-612, 2011

26. Lackner P, Beer R, Broessner G, Helbok R, Galiano K, Pleifer C, et al: Efficacy of silver nanoparticles-impregnated external ventricular drain catheters in patients with acute occlusive hydrocephalus. Neurocrit Care 8:360-365, 2008

27. Lajcak M, Heidecke V, Haude KH, Rainov NG: Infection rates of external ventricular drains are reduced by the use of silver-impregnated catheters. Acta Neurochir (Wien) 155:875-881, 2013

28. Lane JD, Mugamba J, Ssenyonga P, Warf BC: Effectiveness of the Bactiseal Universal Shunt for reducing shunt infection in a sub-Saharan African context: a retrospective cohort study in 160 Ugandan children. J Neurosurg Pediatr 13:140-144, 2014

29. Lemcke J, Depner F, Meier U: The impact of silver nanoparticle-coated and antibiotic-impregnated external ventricular drainage catheters on the risk of infections: a clinical comparison of 95 patients. Acta Neurochir Suppl 114:347-350, 2012

30. Mikhaylov Y, Wilson TJ, Rajajee V, Thompson BG, Maher CO, Sullivan SE, et al: Efficacy of antibiotic-impregnated external ventricular drains in reducing ventriculostomy-associated infections. J Clin Neurosci 21:765-768, 2014

31. Muttaiyah S, Ritchie S, John S, Mee E, Roberts S: Efficacy of antibiotic-impregnated external ventricular drain catheters. J Clin Neurosci 17:296-298, 2010

32. Park YS, Park IS, Park KB, Lee CH, Hwang SH, Han JW: 
Laparotomy versus laparoscopic placement of distal catheter in ventriculoperitoneal shunt procedure. J Korean Neurosurg Soc 48:325-329, 2010

33. Parker SL, Anderson WN, Lilienfeld S, Megerian JT, McGirt MJ: Cerebrospinal shunt infection in patients receiving antibiotic-impregnated versus standard shunts. J Neurosurg Pediatr 8:259-265, 2011

34. Parker SL, Attenello FJ, Sciubba DM, Garces-Ambrossi GL, Ahn E, Weingart J, et al: Comparison of shunt infection incidence in high-risk subgroups receiving antibiotic-impregnated versus standard shunts. Childs Nerv Syst 25:77-85, 2009

35. Pattavilakom A, Xenos C, Bradfield O, Danks RA: Reduction in shunt infection using antibiotic impregnated CSF shunt catheters: an Australian prospective study. J Clin Neurosci 14:526-531, 2007

36. Pople I, Poon W, Assaker R, Mathieu D, Iantosca M, Wang E, et al: Comparison of infection rate with the use of antibioticimpregnated vs standard extraventricular drainage devices: a prospective, randomized controlled trial. Neurosurgery 71:6-13, 2012

37. Ratilal B, Costa J, Sampaio C: Antibiotic prophylaxis for surgical introduction of intracranial ventricular shunts: a systematic review. J Neurosurg Pediatr 1:48-56, 2008

38. Rehman AU, Rehman TU, Bashir HH, Gupta V: A simple method to reduce infection of ventriculoperitoneal shunts. $\mathbf{J}$ Neurosurg Pediatr 5:569-572, 2010

39. Richards HK, Seeley HM, Pickard JD: Efficacy of antibioticimpregnated shunt catheters in reducing shunt infection: data from the United Kingdom Shunt Registry. J Neurosurg Pediatr 4:389-393, 2009

40. Ritz R, Roser F, Morgalla M, Dietz K, Tatagiba M, Will BE: Do antibiotic-impregnated shunts in hydrocephalus therapy reduce the risk of infection? An observational study in 258 patients. BMC Infect Dis 7:38, 2007

41. Rivero-Garvía M, Márquez-Rivas J, Jiménez-Mejías ME, Neth O, Rueda-Torres AB: Reduction in external ventricular drain infection rate. Impact of a minimal handling protocol and antibiotic-impregnated catheters. Acta Neurochir (Wien) 153:647-651, 2011

42. Rozzelle CJ, Leonardo J, Li V: Antimicrobial suture wound closure for cerebrospinal fluid shunt surgery: a prospective, double-blinded, randomized controlled trial. J Neurosurg Pediatr 2:111-117, 2008

43. Steinbok P, Milner R, Agrawal D, Farace E, Leung GK, Ng I, et al: A multicenter multinational registry for assessing ventriculoperitoneal shunt infections for hydrocephalus. Neurosurgery 67:1303-1310, 2010

44. Tamburrini G, Massimi L, Caldarelli M, Di Rocco C: Antibiotic impregnated external ventricular drainage and third ventriculostomy in the management of hydrocephalus associated with posterior cranial fossa tumours. Acta Neurochir (Wien) 150:1049-1056, 2008

45. Theophilus SC, Adnan JS: A randomised control trial on the use of topical methicillin in reducing post-operative ventriculoperitoneal shunt infection. Malays J Med Sci 18:30-37, 2011

46. Thomas R, Lee S, Patole S, Rao S: Antibiotic-impregnated catheters for the prevention of CSF shunt infections: a systematic review and meta-analysis. Br J Neurosurg 26:175184,2012

47. Vardakas KZ, Michalopoulos A, Falagas ME: Fluconazole versus itraconazole for antifungal prophylaxis in neutropenic patients with haematological malignancies: a meta-analysis of randomised-controlled trials. Br J Haematol 131:22-28, 2005
48. Vardakas KZ, Samonis G, Michalopoulos A, Soteriades ES, Falagas ME: Antifungal prophylaxis with azoles in highrisk, surgical intensive care unit patients: a meta-analysis of randomized, placebo-controlled trials. Crit Care Med 34:1216-1224, 2006

49. Walters BC, Hoffman HJ, Hendrick EB, Humphreys RP: Cerebrospinal fluid shunt infection. Influences on initial management and subsequent outcome. J Neurosurg 60:10141021,1984

50. Wang X, Dong Y, Qi XQ, Li YM, Huang CG, Hou LJ: Clinical review: Efficacy of antimicrobial-impregnated catheters in external ventricular drainage - a systematic review and meta-analysis. Crit Care 17:234, 2013

51. Wells GA, Shea B, O'Connell D, Peterson J, Welch V, Losos $M$, et al: The Newcastle-Ottawa Scale (NOS) for assessing the quality of nonrandomised studies in meta-analyses. (http://www.medicine.mcgill.ca/rtamblyn/Readings/The\%20 Newcastle\%20-\%20Scale\%20for\%20assessing \%20the $\% 20$ quality $\% 20$ of $\% 20$ nonrandomised $\% 20$ studies $\% 20$ in $\% 20$ metaanalyses.pdf) [Accessed December 21, 2014]

52. Winkler KM, Woernle CM, Seule M, Held U, Bernays RL, Keller E: Antibiotic-impregnated versus silver-bearing external ventricular drainage catheters: preliminary results in a randomized controlled trial. Neurocrit Care 18:161-165, 2013

53. Woernle CM, Burkhardt JK, Bellut D, Krayenbuehl N, Bertalanffy H: Do iatrogenic factors bias the placement of external ventricular catheters? - a single institute experience and review of the literature. Neurol Med Chir (Tokyo) 51:180186,2011

54. Wong GK, Ip M, Poon WS, Mak CW, Ng RY: Antibioticsimpregnated ventricular catheter versus systemic antibiotics for prevention of nosocomial CSF and non-CSF infections: a prospective randomised clinical trial. J Neurol Neurosurg Psychiatry 81:1064-1067, 2010

55. Wright K, Young P, Brickman C, Sam T, Badjatia N, Pereira $\mathrm{M}$, et al: Rates and determinants of ventriculostomy-related infections during a hospital transition to use of antibioticcoated external ventricular drains. Neurosurg Focus 34(5):E12, 2013

56. Zabramski JM, Whiting D, Darouiche RO, Horner TG, Olson J, Robertson C, et al: Efficacy of antimicrobial-impregnated external ventricular drain catheters: a prospective, randomized, controlled trial. J Neurosurg 98:725-730, 2003

\section{Author Contributions}

Conception and design: Falagas, Konstantelias, Vardakas. Acquisition of data: Konstantelias, Polyzos. Analysis and interpretation of data: all authors. Drafting the article: Konstantelias, Polyzos, Tansarli. Critically revising the article: Vardakas. Reviewed submitted version of manuscript: all authors. Approved the final version of the manuscript on behalf of all authors: Falagas. Statistical analysis: Konstantelias, Vardakas, Tansarli. Administrative/ technical/material support: Falagas. Study supervision: Falagas, Vardakas.

\section{Correspondence}

Matthew E. Falagas, Alfa Institute of Biomedical Sciences, 9 Neapoleos St., 15123 Marousi, Athens, Greece. email: m.falagas@aibs.gr. 


\section{Appendix}

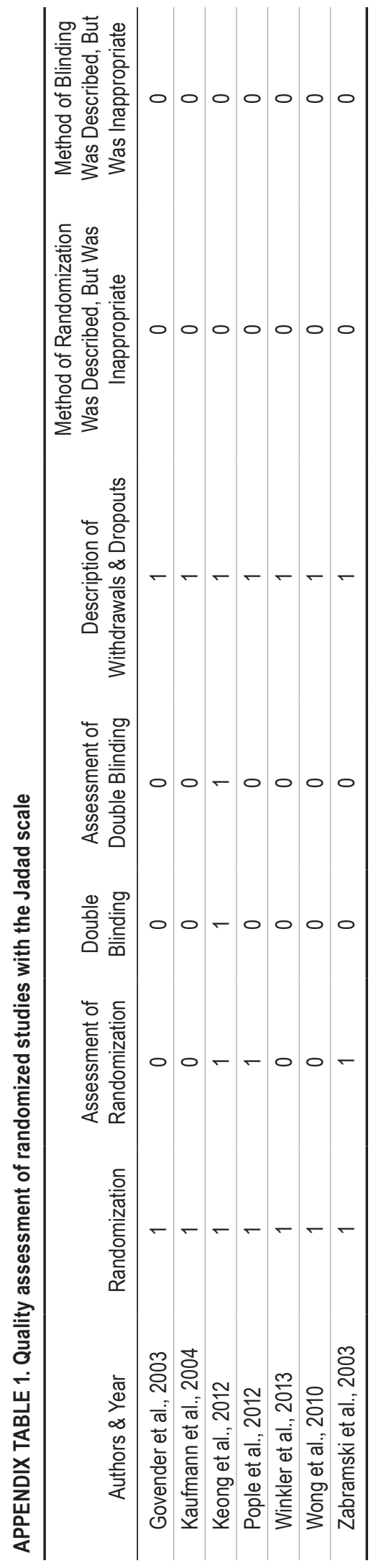




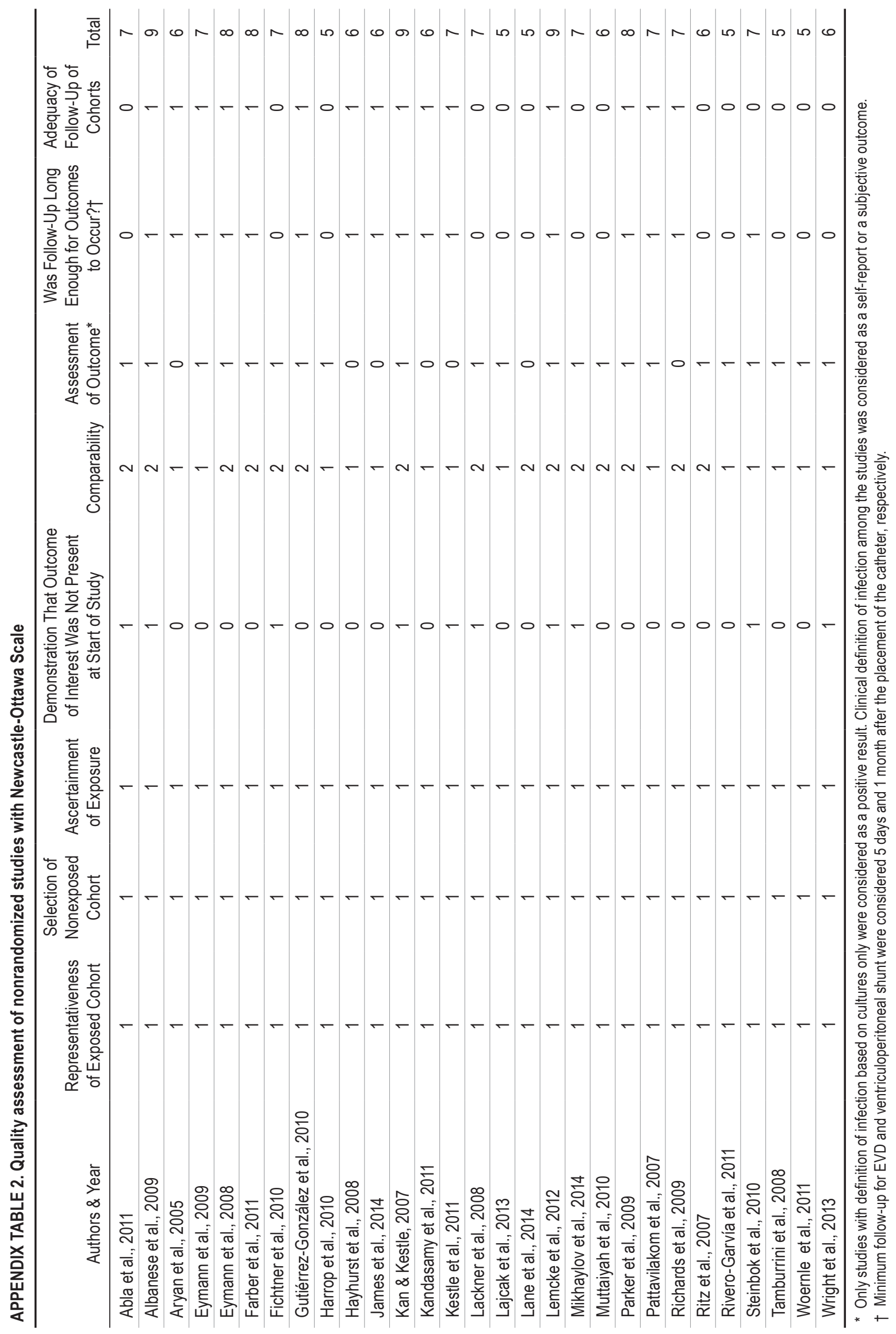




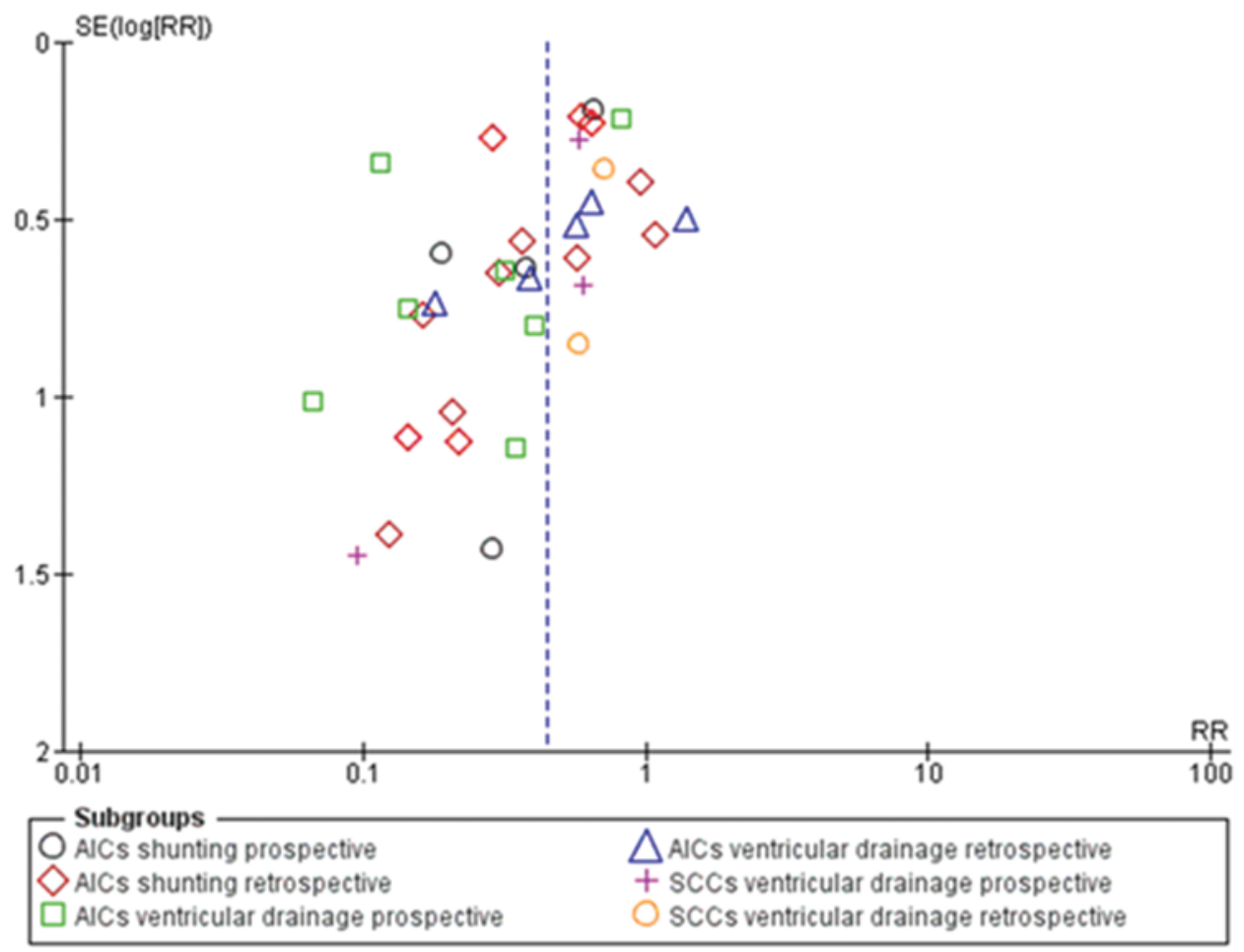

APPENDIX FIG. 1. Funnel plot to assess publication bias. More studies are missing near the middle toward the right, indicating asymmetry. Publication bias in the region of small studies with negative results was observed. SE = standard error. Figure is available in color online only. 